\title{
Netting barriers to prevent mosquito entry into houses in southern Mozambique: a pilot study
}

\author{
Ayubo Kampango ${ }^{1,6^{*}}$, Mauro Bragança ${ }^{2,3}$, Bruno de Sousa $^{2,4}$ and J Derek Charlwood ${ }^{1,5,7}$
}

\begin{abstract}
Background: One of the best ways to control the transmission of malaria is by breaking the vector-human link, either by reducing the effective population size of mosquitoes or avoiding infective bites. Reducing house entry rates in endophagic vectors by obstructing openings is one simple way of achieving this. Mosquito netting has previously been shown to have this effect. More recently different materials that could also be used have come onto the market. Therefore, a pilot study was conducted to investigate the protective effect of three types of material against Anopheles funestus and Anopheles gambiae s.l entry into village houses in Mozambique when applied over the large opening at the gables and both gables and eaves.
\end{abstract}

Methods: A two-step intervention was implemented in which the gable ends of houses (the largest opening) were covered with one of three materials (four year old mosquito bed nets; locally purchased untreated shade cloth or deltamethrin-impregnated shade cloth) followed by covering both gable ends and eaves with material. Four experimental rounds (each of three weeks duration), from four houses randomly assigned to be a control or to receive one of the three intervention materials, were undertaken from March to August 2010 in the village of Furvela in southern Mozambique. Mosquito entry rates were assessed by light-trap collection and the efficacy of the different materials was determined in terms of incidence rate ratio (IRR), obtained through a Generalized Estimating Equations (GEE), of mosquito entry in a treated house compared to the untreated (control) house.

Results: Altogether 9,692 An. funestus and 1,670 An. gambiae s.l. were collected. Houses treated with mosquito netting or the untreated shade cloth had 61.3\% [IRR $=0.39(0.32-0.46) ; P<0.0001]$ and $70 \%$ [IRR $=0.30(0.25-0.37)$; $P<0.001]$ fewer An. funestus in relation to untreated houses, but there was no difference in An. funestus in houses treated with the deltamethrin-impregnated shade cloth [IRR $=0.92(0.76-1.12) ; \mathrm{P}=0.4]$ compared to untreated houses. Houses treated with mosquito netting reduced entry rates of An. gambiae s.l, by $84 \%$ [IRR $=0.16$ $(0.10-0.25) ; P<0.001]$, whilst untreated shade cloth reduced entry rates by $69 \%$ [IRR $=0.31(0.19-0.53) ; P<0.001]$ and entry rates were reduced by $76 \%[\operatorname{IRR}=0.24(0.150 .38) ; P<0.001]$ in houses fitted with deltamethrinimpregnated shade cloth.

Keywords: Netting barriers, Anopheles funestus, Anopheles gambiae s.l, Abundance, House entry rate

\section{Background}

Most malaria transmission occurs inside houses when people are asleep. Improvements to houses were important in reducing malaria transmission in the early part of the last century $[1,2]$. Reducing mosquito entry rates into houses is a simple way of reducing transmission $[3,4]$ and

\footnotetext{
*Correspondence: akampango@gmail.com

'MOZDAN (Mozambican-Danish Rural Malaria Project), PO Box 8,

Morrumbene, Inhambane Province, Mozambique

${ }^{6}$ Present address: Laboratório de Entomologia, Instituto Nacional de Saúde (INS), Av. Eduardo Mondlane, No 1008, Maputo, Mozambique

Full list of author information is available at the end of the article
}

several methods to do this have been proposed, including building houses on stilts [5], using screens [6-9] or blocking the gap between the roof and walls $[10,11]$.

In many rural areas of Africa, houses are simple structures without windows. Hence, mosquitoes, attracted to odour and carbon dioxide, tend to enter such houses via the gap between the roof and the walls [12,13]. This gap, in addition to allowing access to mosquitoes, provides illumination and ventilation and closing the opening with a solid barrier, as recommended by Kirby et al. [11], may not be acceptable in many cases. Airflow may also be increased through other openings if the larger openings

\section{Biomed Central}

(c) 2013 Kampango et al.; licensee BioMed Central Ltd. This is an Open Access article distributed under the terms of the Creative Commons Attribution License (http://creativecommons.org/licenses/by/2.0), which permits unrestricted use, distribution, and reproduction in any medium, provided the original work is properly cited. 
are closed enabling mosquitoes to more easily find these secondary entry points.

An alternative system is to use semi-permeable barriers, such as mosquito netting, that allow air and light to enter the house but which prevent the mosquito from entering. Barriers have been shown to be effective in reducing malaria transmission in Burkina Faso [14] but, with the exception of work in The Gambia [7,9,11], despite the apparent advantages of such a technique, they have not been tested with much rigour elsewhere. Recently developed materials, such as UV-resistant polyethylene shade cloths for the protection of vegetables or vector control may be even more effective or better suited to cover house openings than mosquito netting. A pilot study was, therefore, conducted to determine if, when placed over the openings of houses in a village in southern Mozambique, different types of material of different permeability to airflow, affected mosquito entry rates as measured by light-trap collection. Since houses in this part of Mozambique have a large opening at the gable ends but smaller openings at their sides, the intervention was tested initially by closing only the large openings and subsequently closing all wall to roof openings.

\section{Methods}

\section{Description of study site}

The study was conducted between 24 March 2010 to 30 August 2010 in the village of Furvela $\left(24^{\circ} 43^{\prime} \mathrm{S}, 35^{\circ} 18^{\prime} \mathrm{E}\right)$ in Morrumbene District, Inhambane Province, Mozambique. The village has been described by [15] and [16]. It lies circa $2 \mathrm{~km}$ from a mangrove-bordered coast to the west and is delineated by the Furvela river valley to the north. Both areas provide ample breeding sites for anopheline mosquitoes. A single rainy season occurs from October to March, when approximately 1,200 $\mathrm{mm}$ of rain falls, mostly in February and March. Daily mean temperatures vary between $18^{\circ} \mathrm{C}$ in July and $30^{\circ} \mathrm{C}$ in December. The majority of inhabitants live in rectangular houses built of reed with palm leaf roofs. Most houses do not have windows and have just a single door. There is usually an opening of $15 \mathrm{~cm}$ or more between the end gables and the roof. This provides ventilation, illumination and access for endophilic mosquitoes. The gap between the sides and the roof (the eaves) is smaller or non-existent. Malaria is endemic in the village. Anopheles funestus is the principal vector although Anopheles gambiae, Anopheles arabiensis and Anopheles merus also occur $[15,16]$.

\section{The shade cloths}

Three types of material that differed in their permeability to air were used in the experiment: 1) a deltamethrinimpregnated durable lining (henceforth Zero Vector cloth) manufactured by Vestergaard-Frandsen; 2) a locally purchased untreated-shade cloth used to protect growing vegetables (henceforth Maxixe cloth); and, 3) previously used Interceptor ${ }^{\circledR}$ mosquito bed net (henceforth bed net). The Zero Vector cloth was made of fibres of polyethylene, weighing approximately $55 \mathrm{~g} / \mathrm{sq} \mathrm{m}$. The cloth provides $50 \%$ shading and was impregnated with deltamethrin at $4.4 \mathrm{~g} / \mathrm{kg}$, incorporated into the yarn [17]. The gap size between fibres was circa $1.5 \mathrm{~mm} \times 20 \mathrm{~mm}$ (Figure 1A). The weave of the Maxixe cloth, which was also made of polyethylene, was thicker than Zero Vector cloth and in addition to reducing airflow it reduced the amount of light coming into the house (Figure 1B). The Interceptor ${ }^{\circledR}$ bed net was 75 denier, white polyester treated with the insecticide FENDOZIN ${ }^{\circledR}$, a mixture of the insecticide alphacypermethrin with a binding polymer at a target dose of
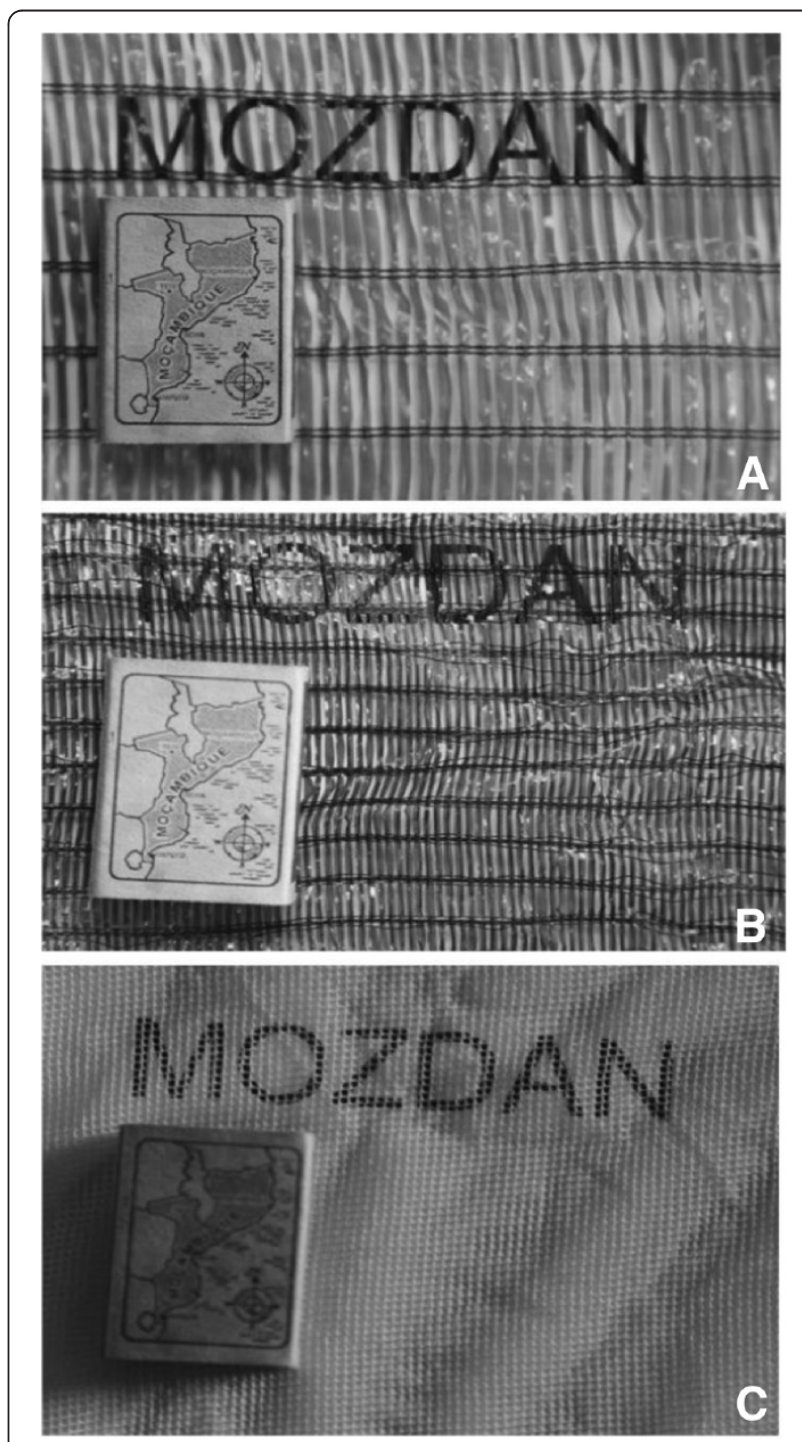

Figure 1 A) Photograph of Zero Vector material used in the experiment. B) Photograph of Maxixe material used in the experiment. C) Photograph of the netting used in the present experiment. 
$6.7 / \mathrm{kg}$ or $200 \mathrm{mg} / \mathrm{m}^{2}$ [18] (Figure $1 \mathrm{C}$ ). The nets had been in use for four years prior to their application in the present experiment.

\section{Airflow measurement}

A CDC light-trap, run from a freshly charged 6v 10 Ah sealed lead acid battery, attached to a $120 \mathrm{~cm}$ long, $15 \times$ $15 \mathrm{~cm}$ cardboard tube was used to provide a constant airflow measured with a calibrated air velocity meter with a resolution of $0.01 \mathrm{~m} / \mathrm{s}$ and an accuracy of $\pm 3 \%$ (TSI TA440). The different fabrics were placed over the end of the opening of the cardboard tube and airflow reduction compared to the flow when no material was in place. For each fabric, airflow was recorded every five seconds over a five-minute period immediately in front and behind the cloth and mean velocities determined. Velocities were also measured between each replicate when no material was obstructing the opening of the funnel. The effect of the different materials on airflow was assessed as percentage reduction in flow. Air flow measurements were normally distributed $(P>0.05)$ therefore, two sample $t$ tests were used to determine the significance of the difference between the mean air flow velocity before and after one the application of the material.

\section{Experimental design}

\section{House selection criteria}

In each of the four experimental rounds four rectangular houses with walls built of reed and roofs of palm leaves, which are the most common form of house in Furvela, were chosen for the experiment. Thus there were 16 houses used in the course of the work. Each experimental round of three weeks duration took place in a different part of the village. Houses used in the experiments were a minimum of $200 \mathrm{~m}$ from each other and at least two people slept in each house.

\section{Application of shade cloth inside houses}

Three of the four houses in each experimental round were randomly assigned to receive one of the interventions: A- Zero Vector; B- Maxixe cloth, or, C- Bed net whilst the fourth served as the control. In the first week of each experimental round and prior to the application of the materials CDC light-traps were run for six consecutive days in each of the houses. In the second week the gable openings of the experimental houses were covered with the designated material (the level of intervention, henceforth designated gables ) [19]. Light-trap collections continued for a further six days, after which, in the third week of the experiment, the relevant material was also applied to the eaves of the treatment houses (the level of intervention henceforth designated gables + eaves). Mosquitoes were again collected for a further six days, giving a total of
18 days of sampling in each house for each experimental round.

\section{Statistical analysis}

Statistical analysis was performed using the software IBM SPSS $^{\circledR}$ v. 20. [20]. Generalized Estimating Equations (GEE) with a negative binomial error distribution, , and a first-order autoregressive correlation structure were used to account, respectively for over dispersion of mosquito counts and serial correlation between repeated catches made in the same house over time [21,22]. Correlation between sequential observation in both An. funestus ( $\mathrm{r}=0.61)$ and An. gambiae sl $(\mathrm{r}=0.54)$, suggests strong serial correlation. The variable 'time' was chosen as subjects and houses as within-subjects factor and GEEs were fitted separately to counts of the two vectors (An. funestus and An. gambiae s.l.) to determine the protective effect of three types of netting material against mosquito entry inside houses, when applied over gables and eaves openings. The efficacy of the material was determined in terms of incidence rate ratio (IRR) of mosquito entry in treated houses compared to the control house [23]. In all the analyses the control house (in which no intervention was in place) was the reference group and a $5 \%$ level was used to determine statistical significance.

\section{Ethical considerations}

The study took place under the aegis of the joint INSDBL project 'Turning houses into traps for mosquitoes' which obtained ethical clearance from the National Bioethics Committee of Mozambique on the $2^{\text {nd }}$ April 2001 (Ref: 056/CNBS/01). Householders were informed about the purpose of the experiment and were told that if they did not like the intervention it would later be removed. Only once consent had been obtained were houses surveyed, the sizes of openings determined and collections inaugurated.

\section{Results}

\section{Airflow data}

Zero Vector cloth had least effect on airflow. This was followed by the mosquito netting, the mean reduction in flow being $16 \%$ and $26 \%$ of the control respectively. The Maxixe cloth, on the other hand, reduced airflow by $87 \%$ (see Table 1).

\section{Mosquito composition and abundance}

A total of 13,639 mosquitoes were caught during the experiment. Anopheles funestus was by far the most abundant species, comprising 71.1\% $(9,692)$ of all mosquitoes collected, followed by An. gambiae s.l. (henceforth called An. gambiae) which comprised $12.2 \%$ of the total. Three hundred and fifty seven (82\%) of the 437 An. gambiae s.l. 


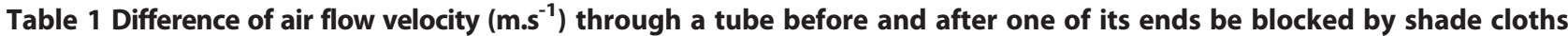

\begin{tabular}{lllll}
\hline & Measurements & Mean air flow $( \pm \mathbf{s e})$ & & \\
\cline { 3 - 4 } Material & $\mathbf{( N )}$ & Without netting & $0.65( \pm 0.003)$ & \\
\hline Bed net & 94 & $0.91( \pm 0.02)$ & $0.048( \pm 0.002)$ & $<$ values \\
Maxixe & 94 & $0.92( \pm 0.004)$ & 0.001 & $<0.001$ \\
Zero vector & 94 & $0.86( \pm 0.006)$ & $0.005)$ & $<0.001$ \\
\hline
\end{tabular}

identified to species from 2002 to 2004 were An. gambiae, 71 were $A n$. arabiensis and nine were An. merus (João Pinto, personal communication). Hence, the majority of the An. gambiae s.l. were An. gambiae s.s. but there was a significant proportion of $A n$. arabiensis in the village. Samples of $A n$. funestus identified to species from 2010 suggested that $A n$. funestus s.s is the only member of the group in the village (James Austin unpub data). Other mosquitoes collected included Culex spp and Anopheles tenebrosus (5.4\% and $4.2 \%$ of the total, respectively). Uranotaenia palmeirimi (2.8\%) and Mansonia africana (2.3\%) were the other species collected in any number.
A considerable variation in the density of mosquitoes collected was observed, both in time and place. Significantly more An. funestus were collected in the final experimental round from the southern area of the village compared to the other three areas $(P<0.001)$, that is: northern, north-eastern and south-eastern areas (Figure 2, Table 1). At the same time this area, had fewer $A n$. gambiae $(\mathrm{P}<0.001)$ than the other areas (Figure 2, Table 2).

Efficacy of materials on mosquito entry

There were three collection days in which the malfunctioning of light-trap in the previous night was noticed

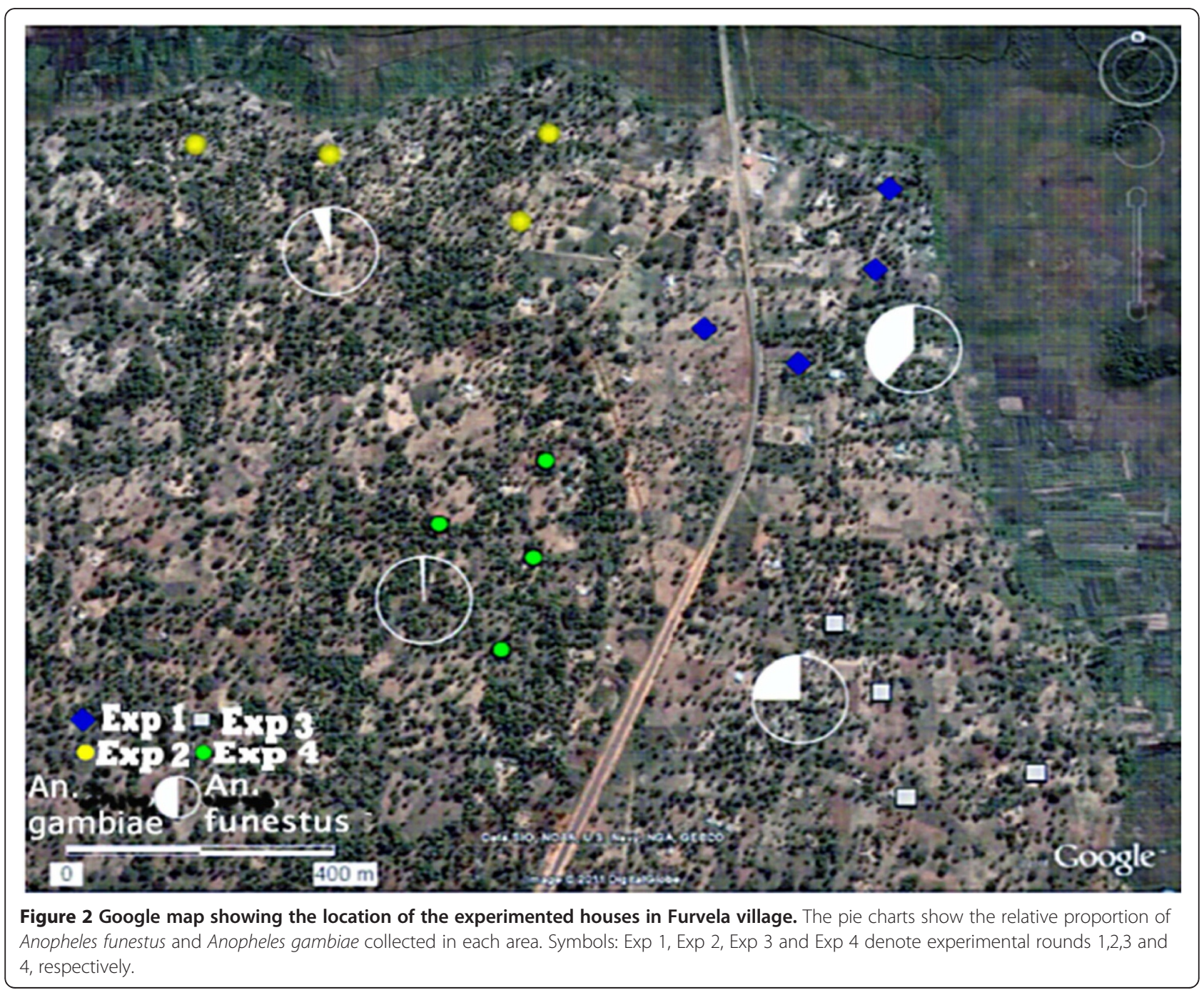


Table 2 Total and mean marginal number (Estimated by GEE model) of Anopheles funestus collected by level of intervention and type of shade cloth; and the efficacy, expressed in terms of incidence rate ratio (IRR) of mosquito entry in treated houses compared to untreated houses, of the materials against the entry of Anopheles funestus into houses in Furvela village

\begin{tabular}{|c|c|c|c|c|}
\hline Factors & Number collected & Mean (95\% Wald CI) & IRR (95\% Wald CI) & $P$ value \\
\hline No intervention & 3874 & $28.9(24.9-33.5)$ & 1 & \\
\hline Gables & 2873 & $21.9(19.4-24.9)$ & $0.75(0.62-0,91)$ & 0.004 \\
\hline Gable and Eaves & 2901 & $23.4(19.8-27.6)$ & $0.8(0.64-1.01)$ & 0.056 \\
\hline No shadecloth & 3372 & $43.4(38.0-49.6)$ & 1 & \\
\hline Zero vector & 3476 & $39.6(34.0-46.0)$ & $0.92(0.76-1.12)$ & 0.40 \\
\hline Maxixe cloth & 1220 & $13.0(10.7-15.7)$ & $0.3(0.25-0.37)$ & $<0.001$ \\
\hline Bed net & 1580 & $16.3(14.4-18.4)$ & $0.39(0.32-0.46)$ & $<0.001$ \\
\hline
\end{tabular}

by the house occupants and reported to a member of the team. Collections from those days were considered as being missing data and were not included in the analysis giving a total of 285 suitable samples instead of the anticipated 288 sampling occasions.

\section{Anopheles funestus}

Table 2 presents the results of the efficacy of three materials on $A n$. funestus entry in treated houses in relation to Control houses. Results suggest that, entry rates of $A n$. funestus were significantly reduced when the material was fitted over the gables of houses and that extending the intervention over eaves did not improve the protective effect. Results indicate that after the intervention was in place there were $70 \%[\operatorname{IRR}=0.30(0.25-0.37) ; \mathrm{P}<0.001]$ and $61.3 \%$ [IRR $=0.39(0.32-0.46) ; \mathrm{P}<0.001]$ fewer $A n$. funestus entering houses treated with Maxixe cloth and mosquito bed net, respectively compared to untreated (control) houses. The number of mosquitoes entering houses treated with Zero Vector was not significantly different to those entering untreated houses [IRR $=0.92$ (0.76-1.12); $\mathrm{P}=0.4]$.

\section{Anopheles gambiae}

All of the materials significantly reduced entry of $A n$. gambiae $(P<0.001)$ (Table 3$)$. There were $84 \%$ fewer $A n$. gambiae $[\mathrm{IRR}=0.16(0.10-0.25) ; \mathrm{P}<0.001]$ inside houses treated with mosquito netting, 76\% [IRR $=0.24 \quad(0.15$ $0.38) ; \mathrm{P}<0.001]$ in houses fitted with Zero Vector and $69 \%($ IRR $=0.31(0.19-0.53) ; \mathrm{P}<0.001)$ in houses treated with Maxixe cloth (Table 3). None of the sixteen households wanted the material removed from their houses at the end of the study.

\section{Discussion}

The results clearly indicate that application of the used mosquito nets or the Maxixe shade cloth significantly reduced the total number of An. funestus entering houses and that all of the materials used in the study reduced the numbers of An. gambiae entering. Houses do, however, have to be relatively well built in the first place and the door must fit securely since gaps around badly fitting doors can easily become entry points for hungry female mosquitoes even when the gables and eaves are covered. In the present experiment the effect of covering only the gable ends or the complete house differed between vectors. Covering the whole house improved the effect against $A n$. gambiae but made no difference to the effect against $A n$. funestus. There are two possible explanations for the enhanced effect against $A n$. gambiae: either the two mosquitoes differ in the way that they enter houses, more An. gambiae going through openings

Table 3 Total and mean marginal number (Estimated by GEE model) of Anopheles gambiae s.l. collected by level of intervention and type of shade cloth and, the efficacy, expressed in terms of incidence rate ratio (IRR) of mosquito entry in treated houses compared to untreated houses, of the material against the entry of Anopheles gambiae into houses in Furvela village

\begin{tabular}{|c|c|c|c|c|}
\hline Factors & Number collected & Mean (95\% Wald Cl) & IRR (95\% Wald CI) & $P$ value \\
\hline No intervention & 1064 & $6.52(4.88-8.72)$ & 1 & \\
\hline Gables & 386 & $1.64(1.23-2.18)$ & $0.17(0.11-0.27)$ & $<0.001$ \\
\hline Gable and Eaves & 220 & $1.12(0.77-1.63)$ & $0.25(0.17-0.37)$ & $<0.001$ \\
\hline No shadecloth & 595 & $6.88(4.98-9.51)$ & 1 & \\
\hline Zero vector & 397 & $1.66(1.18-2.34)$ & $0.24(0.15-0.38)$ & $<0.001$ \\
\hline Maxixe cloth & 458 & $2.13(1.48-3.08)$ & $0.31(0.19-0.50)$ & $<0.001$ \\
\hline Bed net & 220 & $1.12(0.75-1.67)$ & $0.16(0.10-0.25)$ & $<0.001$ \\
\hline
\end{tabular}


in addition to the gables, or the effect had something to do with the insecticide on two of the three materials (Zero Vector and Bed net). The bed nets had been in use for four years and had been washed a number of times so it is not known how much insecticide remained on the material. Since the An. funestus in Furvela are resistant to the pyrethroids used on these materials and resist knockdown for more than 30 minutes when exposed to fresh active ingredient (Charlwood and Kampango, unpublished data) the difference is likely to be due to differing house entry strategies between the two vectors. Unfortunately, the resistance status of the An. gambiae is not known. The efficacy of the one material not impregnated with insecticide also implies that observed reductions were due to physical rather than chemical effects.

Notwithstanding the fact that this was only a pilot study, and for scientific exactitude a much larger randomized control trial would be useful, the results are sufficiently encouraging to suggest to villagers that covering the openings to their houses with netting or shade cloth is a good thing to do. As with bed nets the real problem (whether the material has insecticide or not) is how to get the intervention in widespread use. Although the intervention by itself may not significantly reduce transmission in a village such as Furvela, where inoculation rates are among the highest recorded (Charlwood, unpublished), it is an intervention well worth advocating. It is available, cheap and low-tech and, once in place, does not require further input from the householder. The use of old mosquito nets for this purpose would seem apposite since they are the most readily available material throughout much of the tropics and can be installed with a few nails or wire on a 'do it yourself' basis. Their use would also solve the problem of what to do with old, no longer used, nets.

\section{Competing interests}

The authors declare that they have no competing interests.

\section{Authors' contributions}

AK did the fieldwork, analysed the data and helped write the paper. MF and BdS helped design the study, helped analyse the data, and helped write the paper. JDC designed the study and wrote the paper. All authors read and approved the final manuscript.

\section{Acknowledgements}

We are indebted to the villagers of Furvela who accepted our intervention to work in their houses, and the MOZDAN Project laboratory technicians for their help with the fieldwork. We also thank the reviewers whose comments helped us improve the manuscript. A Bill \& Melinda Gates Foundation Grand Challenge Project financed the study.

\section{Author details}

'MOZDAN (Mozambican-Danish Rural Malaria Project), PO Box 8, Morrumbene, Inhambane Province, Mozambique. ${ }^{2}$ Centro de Malaria e Doenças Tropicais, Lisbon, Portugal. ${ }^{3}$ Universidade Lusofona da Humanidade e Tecnologia, Campo Grande 376, Lisbon, Portugal. ${ }^{4}$ Faculdade de Psicologia e Ciências da Educação, Universidade de Coimbra, Coimbra, Portugal. ${ }^{5} \mathrm{DBL}$ Centre for Health, Research and Development, University of Copenhagen, Fredriksberg, Denmark. ${ }^{6}$ Present address: Laboratório de Entomologia,
Instituto Nacional de Saúde (INS), Av. Eduardo Mondlane, No 1008, Maputo, Mozambique. ${ }^{7}$ Present address: Liverpool School of Tropical Medicine, Pembroke Place, Liverpool L3 5QA, UK.

Received: 3 January 2013 Accepted: 9 March 2013

Published: 16 March 2013

\section{References}

1. Celli A: The new preventative treatment of malaria in Latium. In Collected papers on malaria Angelo Celli, 1899-1912. London: London School of Hygiene \& Tropical Medicine; 1901:1-12.

2. Boyd MF: The influence of obstacles unconsciously erected against Anophelines (housing and screening) upon the incidence of malaria. Am J Trop Med Hyg 1926, 2:157-160.

3. Lindsay SW, Emerson PM, Charlwood JD: Reducing malaria by mosquitoproofing houses. Trends Parasitol 2002, 18:510-514.

4. Lindsay SW, Jawara M, Paine K, Pinder M, Walraven GEL, Emerson PL: Changes in house design reduce exposure to malaria mosquitoes. Trop Med Int Health 2003, 8:512-517.

5. Charlwood JD, Pinto J, Ferrara PR, Sousa CA, Ferreira C, Gil V, Do Rosário V: Raised houses reduce mosquito bites. Malar J 2003, 2:45.

6. Kirby MJ, Bah P, Jones COH, Kelly AH, Jasseh M, Lindsay SW: Social acceptability and durability of two different house screening interventions against exposure to malaria vectors, Plasmodium falciparum infection, and anaemia in children in the Gambia, West Africa. Am J Trop Med Hyg 2010, 83:965-972.

7. Kirby MJ, Ameh D, Bottomley C, Green C, Jawara M, Milligan PM, Snell PC, Conway DJ, Lindsay SW: Effect of two different house-screening interventions on exposure to malaria vectors and on anaemia in children in The Gambia: a randomised control trial. Lancet 2009, 6736:61078.

8. Ogoma S, Kannady K, Sikulu M, Chaki P, Govella N, Mukabana W, Killeen GF: Window screening, ceilings and closed eaves as sustainable ways to control malaria in Dar-es-Salaam. Tanzania Malar J 2009, 8:221.

9. Kirby MJ, Milligan PJ, Conway D, Lindsay SW: Study protocol for a threearmed randomized controlled trial to assess whether house screening can reduce exposure to malaria vectors and reduce malaria transmission in The Gambia. Trials 2008, 9:33.

10. Ogoma SB, Lweitoijera DW, Ngonyani H, Furer B, Russell TL, Mukabana WR, Killeen GF, Moore SJ: Screening mosquito house entry points as a potential method for integrated control of endophagic filariasis, arbovirus and malaria vectors. PLoS Negl Trop Dis 2010, 4:e773.

11. Kirby MJ, Green C, Milligan PM, Sismanidis C, Jasseh M, Conway DJ, Lindsay SW: Risk factors for house-entry by malaria vectors in a rural town and satellite villages in The Gambia. Malar J 2008, 7:2.

12. Snow WF: Studies on the house-entering habits of mosquitoes in The Gambia, West Africa: experiments with prefabricated huts with varied wall apertures. Med Vet Entomol 1987, 1:9-21.

13. Lindsay SW, Snow RW: The trouble with eaves: house entry by vectors of malaria. Trans R Soc Trop Med Hyg 1988, 82:645-646.

14. Majori G, Sabatinelli G, Coluzzi M: Efficacy of permethrin-impregnated curtains for malaria vector control. Med Vet Entomol 1987, 1:185-192.

15. Charlwood JD, Thompson R, Madsen H: Observation on the swarming and mating behaviour of Anopheles funestus from Southern Mozambique. Malar J 2003, 2:2.

16. Kampango A, Cuamba N, Charlwood JD: Does moonlight influence the biting behaviour of Anopheles funestus? Med Vet Entomol 2011, 25:240-246.

17. Vestergaard Frandsen India Private Ltd - Zero vector Durable Lining Specifications. http://www.indiamart.com/vf-india-pvtltd/products. html\#zerovector-durable-lining-specifications.

18. World Health Organization: Specifications and evaluation for public health pesticides-alphacypermethrin for long lasting (coated onto filaments) insecticidal nets. Geneva: World Health Organization; 2009.

19. House screen for mosquito control in Mozambique. http://www.youtube.com/ watch?v=AoNgXsVazQ8.

20. IBM SPSS Statistical Package for Social Sciences. 233 South Wacker Drive, 11thFloor Chicago, IL. USA: SPSS Inc; 2011.

21. Zurr AF, Jeno EN, Saveliev AA, Smith GM: Mixed effects models and extensions in ecology. New York: Springer; 2010. 
22. Ziegler $\mathrm{A}$, Vens $\mathrm{M}$ : Generalized estimating equations: notes on the choice of the working correlation matrix. Methods Inf Med 2010, 5:421-425.

23. Mng'ong'O FC, Sambali JJ, Sabas E, Rubanga J, Magoma J, Ntamatungiro AJ, Turner EL, Nyogea D, Ensink JHJ, Moore SJ: Repellent plants provide affordable natural screening to prevent mosquito house entry in tropical rural settings - results from a pilot efficacy study. PLoS One 2011, 6:e25927.

doi:10.1186/1475-2875-12-99

Cite this article as: Kampango et al: Netting barriers to prevent mosquito entry into houses in southern Mozambique: a pilot study. Malaria Journal 2013 12:99.

\section{Submit your next manuscript to BioMed Central and take full advantage of:}

- Convenient online submission

- Thorough peer review

- No space constraints or color figure charges

- Immediate publication on acceptance

- Inclusion in PubMed, CAS, Scopus and Google Scholar

- Research which is freely available for redistribution 